\title{
Development of deformable mirror composed of piezoelectric thin films for adaptive optics
}

\author{
AUTHOR(S): \\ Kanno, I; Kunisawa, T; Suzuki, T; Kotera, H
}

\section{CITATION:}

Kanno, I ... [et al]. Development of deformable mirror composed of piezoelectric thin films for adaptive optics. IEEE JOURNAL OF SELECTED TOPICS IN QUANTUM ELECTRONICS 2007, 13(2): 155-161

\section{ISSUE DATE:}

2007

URL:

http://hdl.handle.net/2433/50134

\section{RIGHT:}

(c)2007 IEEE. Personal use of this material is permitted. However, permission to reprint/republish this material for advertising or promotional purposes or for creating new collective works for resale or redistribution to servers or lists, or to reuse any copyrighted component of this work in other works must be obtained from the IEEE. 


\title{
Development of Deformable Mirror Composed of Piezoelectric Thin Films for Adaptive Optics
}

\author{
Isaku Kanno, Associate Member, IEEE, Takaaki Kunisawa, Takaaki Suzuki, Member, IEEE, and Hidetoshi Kotera
}

\begin{abstract}
In this paper, we report a piezoelectric deformable mirror composed of piezoelectric thin films for low-voltage adaptive optics (AO). A 2- $\mu$ m-thick piezoelectric $\mathrm{Pb}(\mathrm{Zr}, \mathrm{Ti}) \mathrm{O}_{3}$ (PZT) film was deposited on a Pt-coated silicon-on-insulator (SOI) substrate, and a diaphragm structure of $15 \mathrm{~mm}$ in diameter was fabricated by etching a Si handle wafer. A 19-element unimorph actuator array was produced on the PZT films with an Al reflective layer over the backside of the diaphragm. Measurements of the displacement profile using a laser Doppler vibrometer demonstrated that a large displacement of approximately $1 \mu \mathrm{m}$ was obtained by applying a voltage of $10 \mathrm{~V}_{\mathrm{pp}}$ on one actuator. To examine the application feasibility of the deformable mirror to $\mathrm{AO}$, we reproduced low-order Zernike modes by calculating the voltage on each individual electrode using an influence function matrix. The measurements demonstrated that the deformable mirror could produce the Zernike modes up to the seventh term. Considering the lowvoltage actuation as well as the capability for miniaturization of the electrode size, deformable mirrors (DMs) actuated by PZT films are desirable for low-cost $\mathrm{AO}$.
\end{abstract}

Index Terms-Adaptive optics (AO), deformable mirror, microelectromechanical systems (MEMS), $\mathrm{Pb}(\mathrm{Zr}, \mathrm{Ti}) \mathrm{O}_{3}(\mathrm{PZT})$ thin film.

\section{INTRODUCTION}

$\mathbf{G}$ ROWING demands of adaptive optics (AO) for the industrial and medical applications have propelled the development of small-size and low-cost deformable mirrors (DMs). AO is a significant technique to compensate optical aberration caused by atmospheric turbulence [1], which has been used in astronomical research to obtain high-resolution images. The AO system consists of a wavefront sensor and corrector, which are connected by a real-time controller. In the AO system, a DM is commonly used as a wavefront corrector by adjusting the deflection of the mirror surface to the aberration. Recently, the application area of AO has been expanded to industrial and medical optics, and in particular, in vivo high-resolution human retinal imaging has attracted attention in AO [2]-[4]. To achieve small and low-cost DMs, microelectromechanical systems (MEMS) DMs have successfully been developed and manufactured as commercial products [5]-[11]. The MEMS DMs are commonly actuated by an electrostatic force, and the design and fabrication of the microactuator system have already been established. However, for practical use, several issues still remain

Manuscript received September 7, 2006; revised February 5, 2007. This work was supported in part by the Center of Excellence for Research and Education on Complex Functional Mechanical Systems (COE) Program of the Ministry of Education, Culture, Sports, Science and Technology, and in part by Kyoto City Collaboration of Regional Entities for the Advancement of Technological Excellence, Japan Science and Technology Agency (JST), Japan.

The authors are with the Department of Micro Engineering, Kyoto University, Kyoto 606-8501, Japan (e-mail: kanno@mech.kyoto-u.ac.jp).

Digital Object Identifier 10.1109/JSTQE.2007.894065 unsolved - the large driving voltage of more than $100 \mathrm{~V}$ and the vibration noise of the mirror membrane owing to its very small thickness. Furthermore, since an electrostatic actuator only generates an attractive force and its magnitude is not large, there is a limitation on the control of the mirror deformation.

On the other hand, large-scale DMs are commonly actuated by bulk piezoelectric actuator arrays, and recently, bimorph DMs have been developed for low-cost AO systems [11]-[14]. A piezoelectric actuator can generate a large force, and its response is very fast. In addition, concave and convex deformation can be generated by just changing the polarity of the applied voltage. In order to produce small piezoelectric DMs, the mirror membrane should be actuated by piezoelectric thin films because of the microfabrication of the piezoelectric actuators. Recently, the DMs actuated by $\mathrm{Pb}(\mathrm{Zr}, \mathrm{Ti}) \mathrm{O}_{3}(\mathrm{PZT})$ thin films have been reported [15]. However, these DMs are used in space telescopes with a large membrane and are not aimed at the low-cost commercial products. In this study, to realize small, low-cost DMs, especially for human retinal imaging, we fabricated simple MEMS DMs actuated by piezoelectric PZT thin films. The continuous mirror membrane is deformed by a piezoelectric unimorph microactuator array. To simplify the microfabrication process, the PZT film is deposited on a silicon on insulator (SOI) substrate and the mirror surface of the DMs is prepared on the backside of the diaphragm. To examine the application feasibility of the DMs to $\mathrm{AO}$, we tried to reproduce low-order Zernike modes on the mirror surface, and we discuss actuator performance of the DM for AO.

\section{PIEZOELECTRIC MEMS DM DESIGN}

\section{A. Structure of Piezoelectric Microactuator}

The piezoelectric MEMS DM consists of a continuous membrane mirror on the backside of which piezoelectric PZT films are deposited. The piezoelectric actuator array consists of a unimorph structure of PZT film and an Si device layer. The bottom electrode is prepared as a common electrode at the interface of the PZT film and Si, while 19 individual electrodes are arranged on PZT. The individual electrodes are laid out as segments of concentric circles. The diameter of the center circle and width of the second ring electrodes are $2 \mathrm{~mm}$, and the width of the outer electrode is $3 \mathrm{~mm}$ [Fig. 1(a)]. Application of the voltage along with the film thickness induces a transverse strain, which is converted into the vertical deflection due to the restriction of the Si elastic layer. Both concave and convex deformations are possible by just changing the polarity of the applied voltage. The electrode pattern corresponded to the Zernike mode and is of the same design as in bimorph DMs [12], [16]. The plan and 
(a)

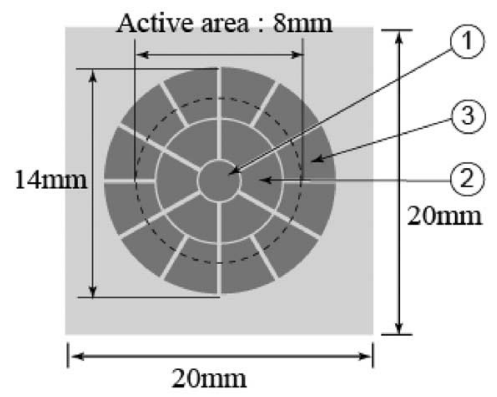

(b)

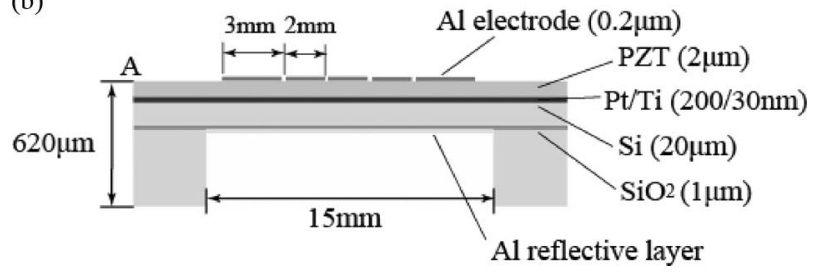

Fig. 1. Schematic illustration of the piezoelectric MEMS DMs. (a) Plane view of the piezoelectric actuator array. The segmented ring electrodes are arranged on the PZT film and the displacement profiles are examined on the actuators (1), (2), and (3) as indicated in the figure. The mirror surface is prepared on the backside of the membrane. (b) Cross-sectional structure of the mirror. Diameter of the membrane is $15 \mathrm{~mm}$, whereas the active mirror area is 8 - $\mathrm{mm}$ circular plane.

cross-sectional structure of the piezoelectric MEMS DM is illustrated in Fig. 1. The thickness of the PZT and Si membrane is 2 and $20 \mu \mathrm{m}$, respectively. Compared to the conventional electrostatic MEMS DMs, the thickness of the membrane can be greater because the piezoelectric force is generally larger than the electrostatic one. Therefore, the membrane of the piezoelectric DM is stable even in the presence of noise and disturbance from external vibrations. In the piezoelectric DM of this study, the diameter of the membrane is $15 \mathrm{~mm}$, whereas the active area for the correction of aberration is $8 \mathrm{~mm}$ in diameter.

\section{B. Finite-Element Method (FEM) Simulation}

An FEM simulation was carried out using commercial software MARC to estimate the displacement profile of the DM. To simplify the simulation model, we only consider the PZT/Si bilayer, and neglect the mechanical effects of electrodes and the buried $\mathrm{SiO}_{2}$ layer. For the material properties of the PZT layer, we use typical bulk values of PZT; Young's modulus and piezoelectric coefficient $e_{31}$ are $70 \mathrm{GPa}$ and $-6.5 \mathrm{C} / \mathrm{m}^{2}$, respectively. For the $20-\mu \mathrm{m}$-thick Si layer, anisotropic mechanical compliance of the single Si crystal is used [17]. The positions of the electrode for the calculation and resulting 3-D and 2-D displacement profiles are shown in Fig. 2. The displacement profiles of the mirror are calculated using a half model of the membrane under the application of a voltage of $1 \mathrm{~V}_{\mathrm{pp}}$ on each actuator. The simulation demonstrated that the maximum displacement of each actuator is approximately $0.4 \mu \mathrm{m}$. Assuming that the displacement is proportional to the applied voltage, the maximum displacement reaches $4 \mu \mathrm{m}$ when $10 \mathrm{~V}_{\mathrm{pp}}$ is applied to one actuator. This result indicates that the PZT thin-film actuator can generate large displacements with low operating voltages, be- (a)
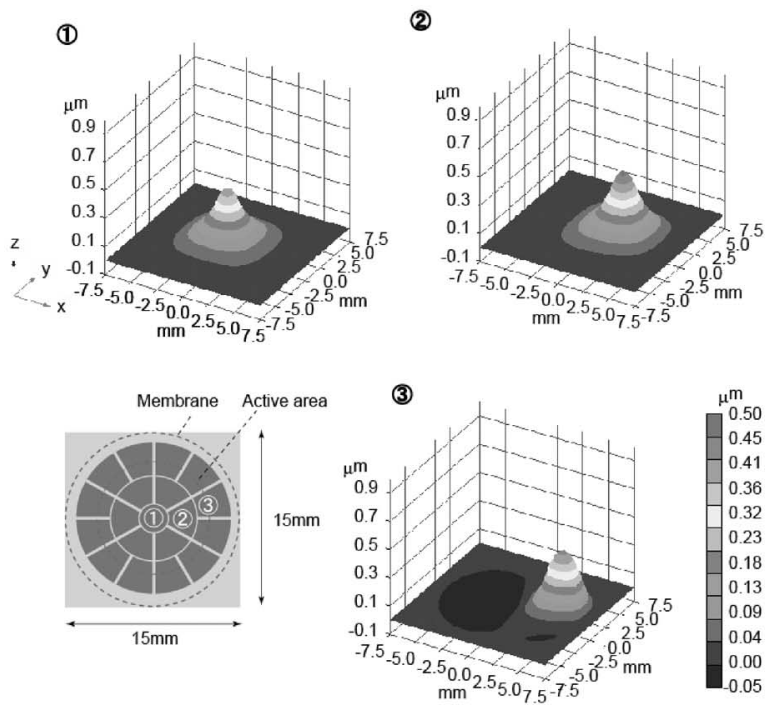

(b)

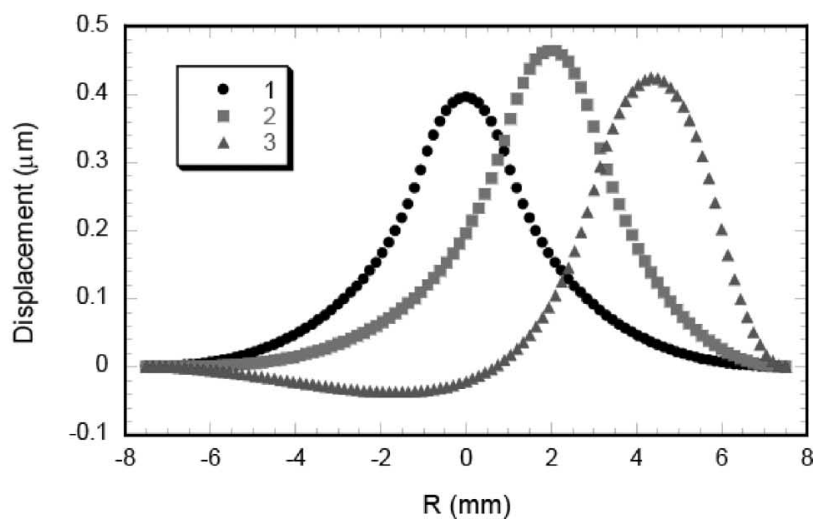

Fig. 2. Displacement profiles of the actuators for a voltage of $1 \mathrm{~V}$. (a) 3-D displacement profiles of $15-\mathrm{mm}^{2}$ area on the actuators (1), (2), and (3). (b) 2-D displacement profiles along the diameter of the membrane. All actuators have a displacement of approximately $0.4 \mu \mathrm{m}$ at $1 \mathrm{~V}$.

cause the small thickness of the PZT film increases the internal electric field. The displacement of the center electrode 1 shows a symmetrical profile, as shown in Fig. 2. However, for the outer electrode 3 , the displacement profile is asymmetrical, and the symmetrical position of actuator 3 deflects in the opposite direction, as shown in Fig. 2. The asymmetrical displacement profile of the outer electrode is caused by the asymmetrical binding condition around actuator 3. However, the displacemnet in the opposite direction in the symmetrical position is so small that this effect is trivial for the practical control of the deformation of the mirror.

\section{FABRICATION OF PIEZOELECTRIC MEMS DM}

Piezoelectric MEMS DMs were fabricated according to the design of the diaphragm structure, as illustrated in Fig. 1, and the fabrication process is illustrated in Fig. 3. First, a 2- $\mu \mathrm{m}-$ thick piezoelectric PZT film was deposited on a Pt/Ti-coated SOI substrate using RF sputtering. In this paper, we use a SOI 
(1)

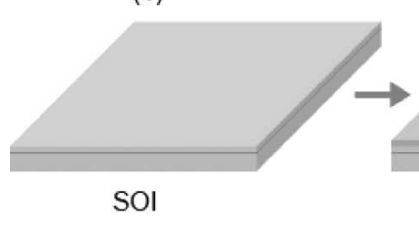

(2) Ti sputtering

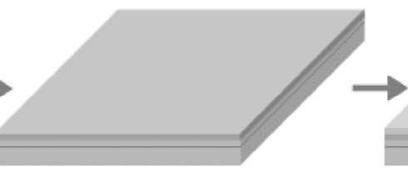

$\mathrm{Ti} / \mathrm{SOI}$
(3) Pt sputter

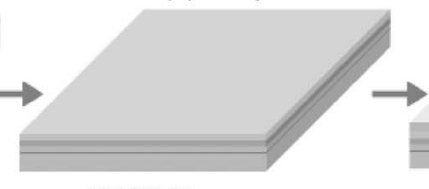

$\mathrm{Pt} / \mathrm{Ti} / \mathrm{SOI}$
(4) PZT sputter

(5) Lift-off resist pattterning

(6) Al evaporation

(7) Al patterning

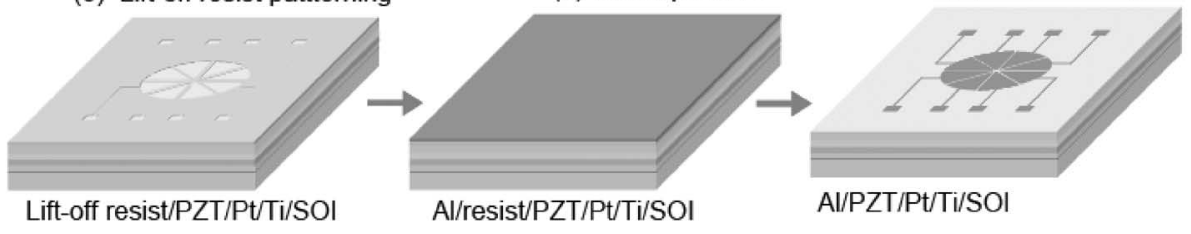

(8) Dry Film patterning

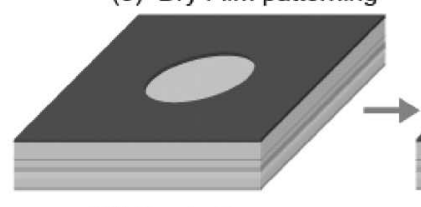

(9) Si patterning
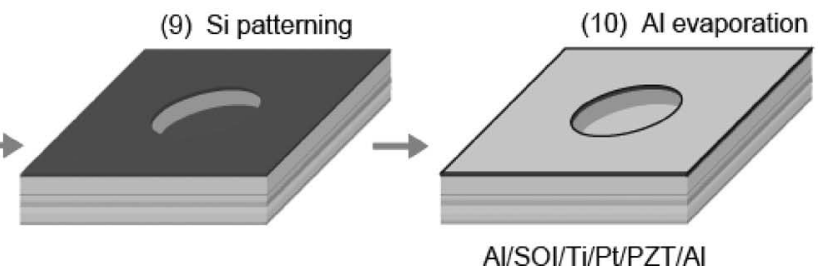

SOI backside

(a)

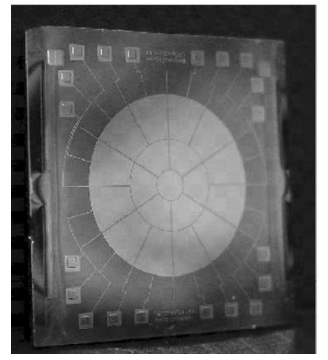

(b)

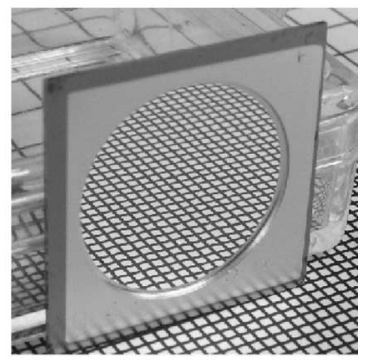

Fig. 3. Fabrication process of the piezoelectric MEMS DMs. The PZT film was deposited on a SOI wafer and Al individual electrodes were prepared on it.Successively, backside of the wafer was etched out into a diaphragm structure. The BOX layer acted as a stopping layer for RIE etching, and finally, Al reflective layer was deposited over the membrane.

wafer as a substrate for PZT deposition such that a Si membrane of homogeneous thickness can easily be obtained by just etching out the Si handle wafer. After the deposition of the PZT films, individual $\mathrm{Al}$ electrodes were deposited by a thermal evaporator and patterned by the liftoff process. Successively, the diaphragm structure was prepared by etching the $\mathrm{Si}$ handle wafer using $\mathrm{SF}_{6}$ plasma. As a photoresist for Si etching, we used a 30- $\mu$ m-thick dry film to maintain the plasma process over a long period. A dry film is a thick photosensitive film usually used for patterning of printed circuit boards. First, the dryfilm (DuPont MRC Dryfilm: SA130) was laminated on the backside of the substrate, and ultraviolet light was directed through the photomask onto it. After developing in the alkaline developer, the diaphragm structure was prepared by etching the $\mathrm{Si}$ handle wafer using $\mathrm{SF}_{6}$ plasma.

The buried oxide (BOX) layer acts as a stopping layer for the reactive-ion etching (RIE) using $\mathrm{SF}_{6}$, and the exposed BOX layer has a very smooth surface, which is used as a mirror surface after the deposition of the Al film. Photographs of the DM produced are shown in Fig. 4.

The flatness of the mirror surface was measured with a white light interferometer (Wyko NT-1100), and the result is shown in Fig. 5. The measurement revealed that the peak-to-valley height over the whole membrane is more than $5 \mu \mathrm{m}$, whereas that within the active area is approximately $2 \mu \mathrm{m}$. The stress state of the membrane strongly affects the initial shape as well as the actuation of the mirror. According to the surface profile of the mirror, the initial deformation is mainly due to the residual compressive stresses of the buried $\mathrm{SiO}_{2}$ layer and the individual Al electrodes, because PZT films on Si have a tensile stress. We expect that the initial deformation of the mirror could be
Fig. 4. Photographs of obtained DMs. (a) Actuator side. (b) Mirror side.

improved by optimizing the thickness or residual stresses of the BOX layer and the $\mathrm{Al}$ electrodes.

\section{Characterization OF Deformation}

\section{A. Actuator Performance}

The displacement of the mirror was measured by a laser Doppler vibrometer (LDV) - not only magnitude of the displacement, but also the direction of the displacement by the phase output displacement. Before carrying out the measurements, we applied a voltage of $-30 \mathrm{~V}$ on each electrode as a poling treatment. The application of $-30 \mathrm{~V}$ on the $2-\mu \mathrm{m}$ thick PZT films results in a large electric field of $-150 \mathrm{kV} / \mathrm{cm}$ that is large enough to align in polar direction since the coercive electric field, where the polarization is reversed, was measured to be around $\pm 50 \mathrm{kV} / \mathrm{cm}$ for the sputtered PZT films on $\mathrm{Si}$ [18]. In addition, it is reported that the polarization state of the 


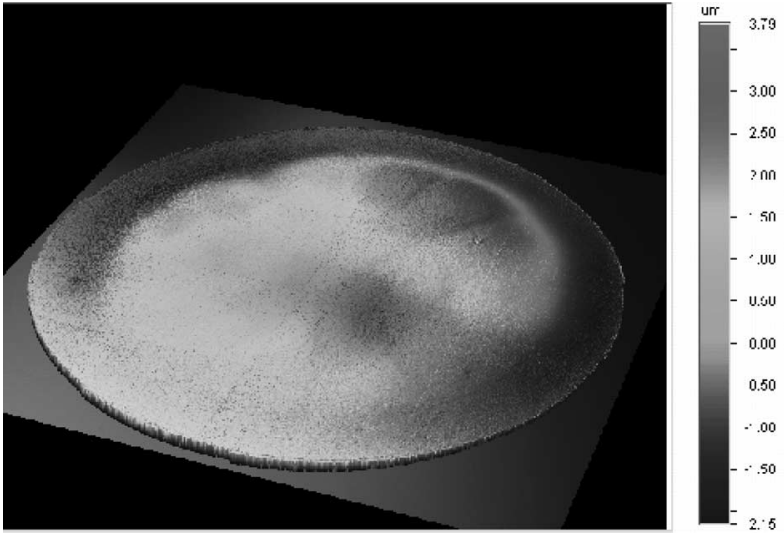

Fig. 5. Initial surface profile of the mirror measured with a white light interferometer. The peak-to-valley roughness of the whole mirror membrane is more than $5 \mu \mathrm{m}$ due to the residual stresses of the $\mathrm{Al}$ individual electrodes.
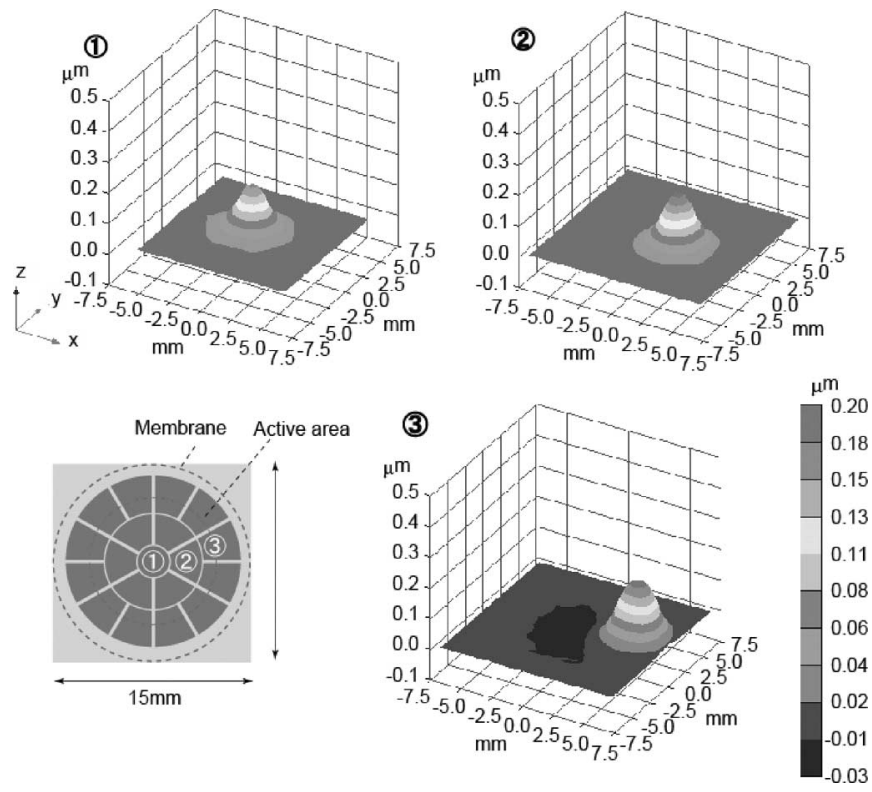

Fig. 6. 3-D displacement profiles of the actuators (1), (2), and (3), applying sine wave signals at $300 \mathrm{~Hz}$. The measurements were conducted using an LDV. The shapes of the displacement profiles are almost similar to those of the FEM results.

sputtered PZT films is more stable than that of sol-gel-derived PZT film [19].

We applied a unipolar sine wave signal of $2 \mathrm{~V}_{\mathrm{pp}}$ at $300 \mathrm{~Hz}$ on three individual actuators, and the 3-D displacement profiles were observed (Fig. 6). The measurements revealed that the displacement profiles are almost the same as those for the FEM simulation. The inner actuators are connected to the outer electrode pads by the $30-\mu \mathrm{m}$-wide Al lines. Since the width of the extraction lines are much smaller than those of actuators, we could not find their effects in Fig. 6.

Fig. 7 shows the maximum displacement of three different actuators as a function of voltage. The displacement increases with increasing applied voltage, and application of $10 \mathrm{~V}$ on one actuator can generate more than $1-\mu \mathrm{m}$ displacement. However, this value is smaller, and is about half of the FEM simulation re-

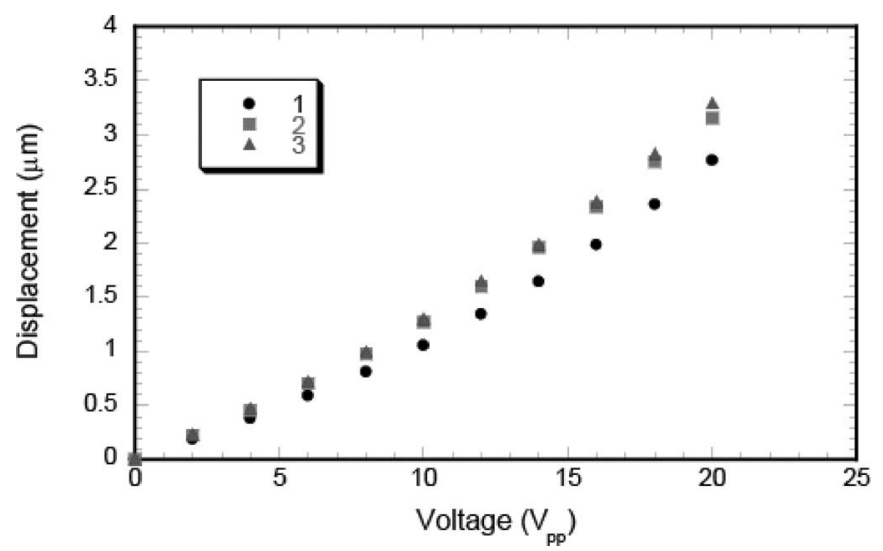

Fig. 7. Maximum displacement as a function of applied voltage on the actuators 1,2 , and 3 . The displacement increases with the voltage, and the application of $10 \mathrm{~V}$ generates more than $1 \mu \mathrm{m}$.

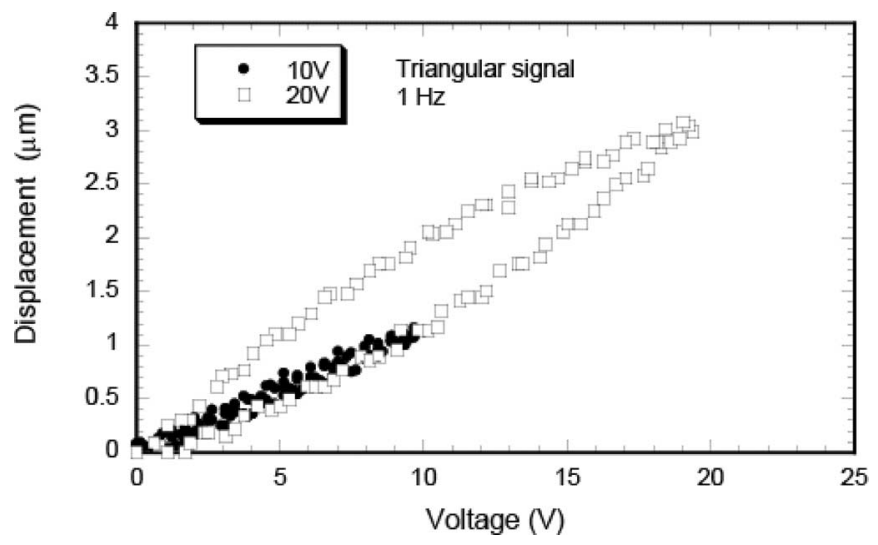

Fig. 8. Displacement hysteresis of the actuator. Triangular signals of 10 or $20 \mathrm{~V}$ were applied on the actuator 1 . The large hysteresis is induced by $20 \mathrm{~V}$, whereas the hysteresis can be improved by low application voltage of $10 \mathrm{~V}$.

sults. The smaller displacement is attributed to the increase in the stiffness of the membrane due to the top and bottom electrodes. Another possible reason is the smaller piezoelectric properties of the PZT films compared with those of the bulk materials. On the other hand, the increasing ratio of the displacement increases with voltage. This phenomenon is related to the domain motion of the PZT films, which also causes hysteresis of the displacement.

For the deformation of the mirror, the maximum operating voltage is limited by the breakdown voltage and the polarization reversal voltage of the PZT film. The breakdown voltage of the $2-\mu \mathrm{m}$-thick PZT films is approximately $100 \mathrm{~V}$, which is the limit of the voltage along polar direction for convex curvature of the mirror. On the other hand, the polarization reversal voltage is around $10 \mathrm{~V}$, which is the maximum voltage of the opposite direction of the polarization to produce concave deformation of the mirror.

The hysteresis of the displacement is measured using a fringe count laser interferometer. We applied unipolar triangular signals of 10 or $20 \mathrm{~V}_{\mathrm{pp}}$ at $1 \mathrm{~Hz}$ to electrode 1 , and hysteresis curves of the displacement are plotted, as shown in Fig. 8. When we apply voltages up to $20 \mathrm{~V}$, large displacement hysteresis occurs. 

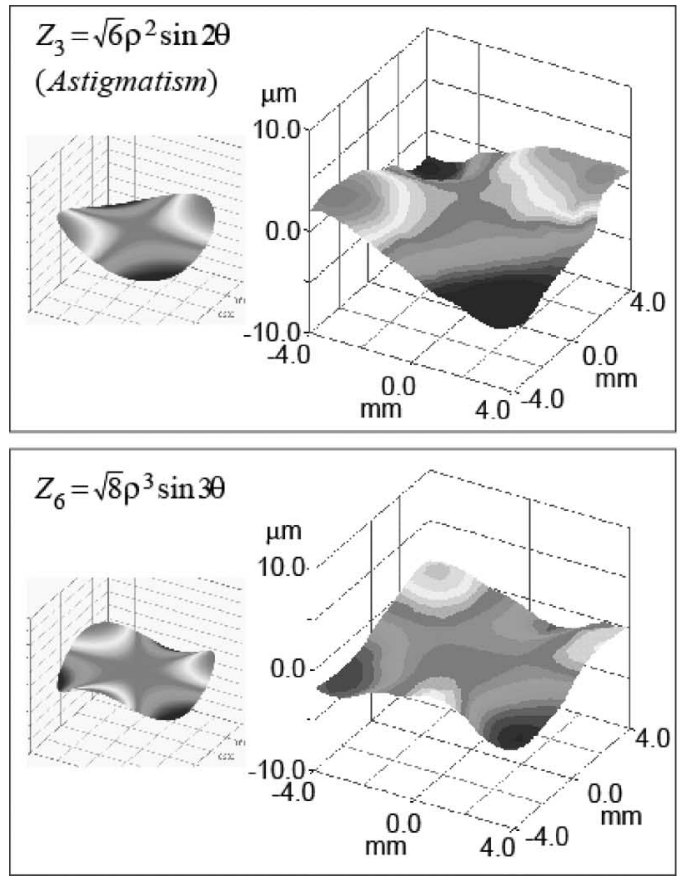
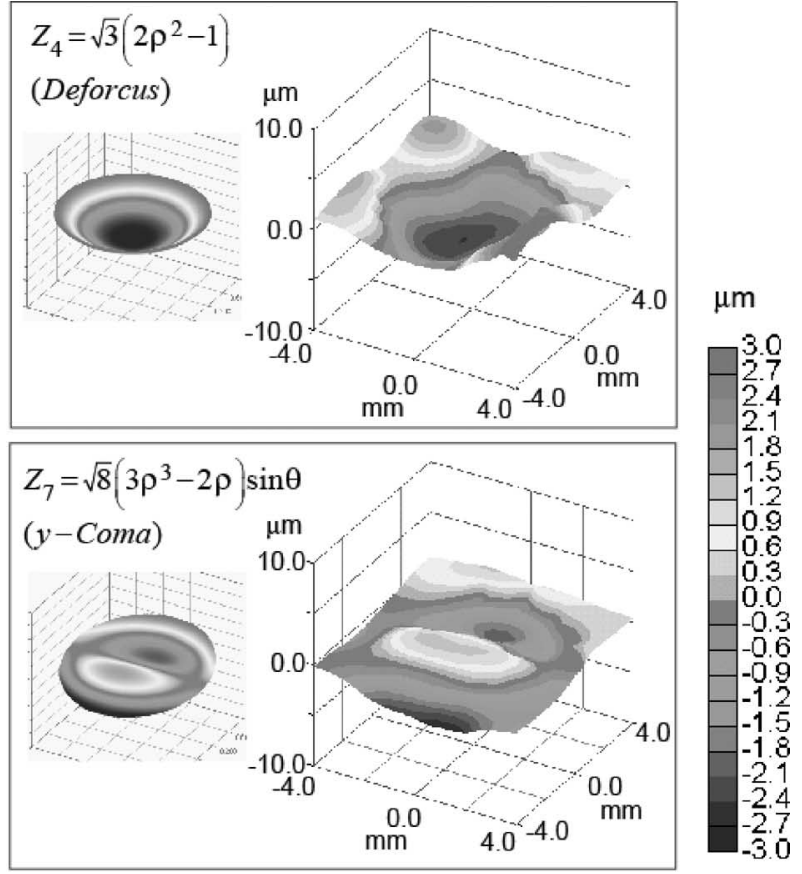

Fig. 9. Ideal and measured displacement profiles of the Zernike modes of third, fourth, sixth, and seventh term. The axes of ideal profiles are arbitrary. There are some deviations between ideal and measured profiles, and we have successively reproduce low-order Zernike modes on the piezoelectric MEMS DMs.

Large hysteresis is also reported in the commercial piezoelectric DMs [11], and this phenomenon originates from the domain rotation of the PZT [18]. On the other hand, for low-voltage operation up to $10 \mathrm{~V}$, the hysteresis is very small and the linear relationship between voltage and displacement is maintained. Although the PZT films on SOI substrates have randomly oriented polycrystalline structures, the low-voltage operation of the DMs can prevent the domain rotation, and an improvement in the deformation controllability would be expected.

\section{B. Reproduction of Zernike Mode}

In order to evaluate the applicability of piezoelectric MEMS DMs to the AO system, we produced deformation of the mirror corresponding to Zernike mode. The Zernike polynomials are commonly used to express the deformation of a circular plane by a complete set of orthogonal polynomials [9]. If the optical wavefront is represented by the Zernike polynomials, aberration can be corrected by producing deformation of the Zernike mode by the DMs.

The circular mirror surface $\Phi(x, y)$ can be expressed as

$$
\Phi(x, y)=\sum_{i=1}^{M} a_{i} Z_{i}(x, y)
$$

where $M$ is the total number of Zernike polynomials, and $Z_{i}$ is the $i$ th Zernike polynomial with a coefficient $a_{i}$. A set of Zernike coefficients is expressed by a vector $a=\left[a_{1}, a_{2}, \ldots, a_{M}\right]^{T}$, where $\mathrm{T}$ means matrix transpose. This means that the vector a uniquely represents the mirror surface. Also, the surface deformation can be controlled by the voltage of the electrodes, and the relationship between the mirror surface and the voltage for the individual electrodes is expressed by influence functions matrix B

$\mathbf{a}=\mathbf{B v}, \quad\left(\begin{array}{c}a_{1} \\ a_{2} \\ \vdots \\ a_{M}\end{array}\right)=\left(\begin{array}{cccc}b_{11} & b_{12} & \cdots & b_{1 N} \\ b_{21} & b_{22} & \cdots & b_{2 N} \\ \vdots & \vdots & \ddots & \vdots \\ b_{M 1} & b_{M 2} & \cdots & b_{\mathrm{MN}}\end{array}\right)\left(\begin{array}{c}v_{1} \\ v_{2} \\ \vdots \\ v_{N}\end{array}\right)$

where $N$ is the number of the electrode and vector $\mathbf{v}$ represents the voltage applied to the piezoelectric actuator. The inverse of the influence functions matrix $\mathbf{B}$ is called the control matrix $\mathbf{B}^{+}$, and the required voltage for the desired surface is obtained by

$$
\mathbf{v}=\mathbf{B}^{+} \mathbf{a} .
$$

In this paper, we evaluated Zernike mode from second to fourth radial order, and thus, we considered the influence function $\mathbf{B}$ of $18 \times 19$ matrix. Since the numbers $M$ and $N$ are different, a pseudoinverse matrix is calculated as the control matrix $\mathbf{B}^{+}$. The influence functions matrix $\mathbf{B}$ is derived from the displacement profiles by applying $10 \mathrm{~V}$ to each actuator. We also tried to reproduce low-order Zernike modes of $Z_{3}, Z_{4}, Z_{6}$, and $Z_{7}$ and observed the deformation characteristics with an LDV. The Zernike modes of $Z_{3}, Z_{4}$, and $Z_{7}$ are known as the aberrations of astigmatism, defocus, and coma-aberration, respectively. The voltage for each electrode is calculated from (3), assuming the displacement of the actuator is proportional to the voltage. In these tests, the applied voltage on each actuator is less than $10 \mathrm{~V}$, and only dynamic displacement profiles were observed without applying bias voltage for planarization of the mirror. Fig. 9 shows both the ideal and observed Zernike modes. Although some deviations still remain between ideal and observed deformations, we could confirm that the piezoelectric 
MEMS DMs can reproduce low-order Zernike modes by lowvoltage operation. For the practical use of the piezoelectric MEMS DMs to AO, the initial surface of the mirror should be flattened by the same procedure of the wavefront correction as mentioned before. In addition, it is necessary to increase the number of individual electrodes in order to correct the higher order aberration. However, this study demonstrated that piezoelectric MEMS DMs composed of PZT films are very attractive devices for the low-voltage wavefront generator in AO.

\section{CONCLUSION}

We have developed piezoelectric MEMS DMs composed of PZT thin films on SOI substrate for AO, and examined the deformation characteristics of the mirror. The continuous mirror membrane consists of a unimorph actuator array with 19 individual electrodes corresponding to Zernike modes. FEM simulation indicates that a large displacement is generated by low-voltage operation due to the small thickness of the piezoelectric PZT films. In the fabrication of the DM, a mirror surface can be easily obtained on the backside of the substrate by the deposition of an $\mathrm{Al}$ reflective layer on an exposed $\mathrm{BOX}$ layer, which acts as a stopping layer for the $\mathrm{SF}_{6}$ RIE. The displacement profiles of the mirror surface were measured by an LDV and were similar to the FEM simulation results. However, the maximum displacements of each actuator are smaller than that of the FEM results owing to the increase in stiffness due to the electrodes as well as low-piezoelectric properties of the PZT thin films. The voltage required for each electrode to generate Zernike modes were calculated by control matrix $\mathbf{B}^{+}$, which was derived from the relationship between applied voltage on each actuator and the displacement profile. The measurement of the displacement profile revealed that the piezoelectric MEMS DM could produce low-order Zernike modes using low voltage.

\section{ACKNOWLEDGMENT}

The authors would like to thank Dr. T. Shirai of Advanced Industrial Science and Technology and S. Inoue of Hamamatsu Photonics K.K. for useful discussions on the characterization of the DMs.

\section{REFERENCES}

[1] J. W. Hardy, J. E. Lefebvre, and C. L. Koliopoulos, "Real-time atmospheric compensation,” J. Opt. Soc. Amer., vol. 67, pp. 360-369, 1977.

[2] J. Liang, D. R. Williams, and D. T. Miller, "Supernormal vision and highresolution retinal imaging through adaptive optics," J. Opt. Soc. Amer. A., vol. 14, pp. 2884-2892, 1997.

[3] H. Hofer, L. Chen, G. Y. Yoon, B. Singer, Y. Yamauchi, and D. R. Williams, "Improvement in retinal image quality with dynamic correction of the eye's aberrations," Opt. Exp., vol. 8, no. 11, pp. 631-643, 2001.

[4] N. Doble, G. Yoon, L. Chen, P. Bierden, B. Singer, S. Olivier, and D. R. Williams, "Use of a microelectromechanical mirror for adaptive optics in the human eye," Opt. Lett., vol. 27, no. 17, pp. 1537-1539, 2002.

[5] G. Vdovin and P. M. Sarro, "Flexible mirror micromachined in silicon," Appl. Opt., vol. 34, no. 16, pp. 2968-2972, 1995.

[6] L. Zhu, P.-C. Sun, D.-U. Bartsch, W. R. Freeman, and Y. Faiman, "Adaptive control of a micromachined continuous-membrane deformable mirror for aberration compensation," Appl. Opt., vol. 38, no. 1, pp. 168-179, 1999.
[7] J. A. Perreault, T. G. Bifano, B. M. Levine, and M. N. Horenstein, "Adaptive optic correction using microelectromechanical deformable mirrors," Opt. Eng., vol. 41, no. 3, pp. 561-566, 2002.

[8] E. J. Fernandez and P. Artal, "Membrane deformable mirror for adaptive optics: performance limits in visual optics," Opt. Exp., vol. 11, no. 9, pp. 1056-1069, 2003.

[9] L. Zhu, P.-C. Sun, D.-U. Bartsch, W. R. Freeman, and Y. Fainman, "Wavefront generation of Zernike polynomial modes with a micromachined membrane deformable mirror," Appl. Opt., vol. 38, no. 28, pp. 60196026, 1999.

[10] D. J. Dagel, W. D. Cowan, O. B. Spahn, G. D. Grossetete, A. J. Grine, M. J. Shaw, P. J. Resnick, and B. Jokiel, "Large-stroke MEMS deformable mirrors for adaptive optics," J. Microelectromech. Syst., vol. 15, no. 3, pp. 572-583, 2006.

[11] E. Dalimier and C. Dainty, "Comparative analysis of deformable mirrors for ocular adaptive optics," Opt. Expr., vol. 13, no. 11, pp. 4275-4285, 2005

[12] D. A. Horsley, H. Park, S. P. Laut, and J. S. Werner, "Characterization for vision science applications of a bimorph deformable mirror using phaseshifting interferometry," Proc. SPIE, vol. 5688, pp. 133-144, 2005.

[13] M. S. Griffith, L. C. Laycock, and N. Archer, "Novel high bandwidth bimorph deformable mirrors," Proc. SPIE, vol. 5614, pp. 109-118, 2004.

[14] S. G. Lipson, E. N. Riback, and C. Schwartz, "Bimorph deformable mirror design," Proc. SPIE, vol. 2201, pp. 703-714, 1994.

[15] Y. Hishinuma and E.-H. Yang, "Piezoelectric unimorph microactuator arrays for single-crystal silicon continuous-membrane deformable mirror," J. Microelectromech. Syst., vol. 15, no. 2, pp. 370-379, 2006.

[16] M. G. Lofdahl. G. Scharmer and W. Wei, "Calibration of a deformable mirror and Strehl ratio measurements by use of phase diversity," Appl. Opt., vol. 39, pp. 94-103, 2000.

[17] M. Madou, Fundamentals of Microfabrication, 2nd ed. Boca Raton, FL: CRC Press, 2002, pp. 195-199.

[18] I. Kanno, H. Kotera, and K. Wasa, "Measurement of transverse piezoelectric properties of PZT thin films," Sens. Actuators A, vol. 107, pp. 68-74, 2003

[19] J. F. Shepard, Jr., F. Chu, I. Kanno, and S. T-. McKinstry, "Characterization and aging response of the $\mathrm{d} 31$ piezoelectric coefficient of lead zirconate titanate thin films," J. Appl. Phys., vol. 85, pp. 6711-6717, 1999.

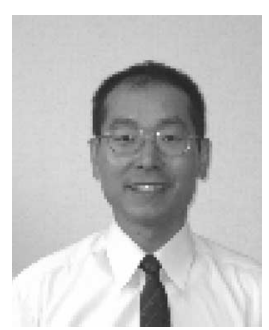

Isaku Kanno (M'02-A'02) was born in Fukuoka, Japan, in 1966. He received the B.S., M.S., and Ph.D. degrees in nuclear engineering from Osaka University, Osaka, Japan, in 1989, 1991, and 1999, respectively.

From 1991 to 2002, he was with the Research and Development Center, Panasonic, Inc., Osaka, Japan. From 2002 to 2005, he was an Associate Professor in the Department of Mechanical Engineering, Kyoto University, Kyoto, Japan, where he is currently an Associate Professor in the Department of Micro Engineering. His current research interests include growth of the ferroelectric thin films, characterization of the relationship between crystalline structure and piezoelectric properties of the films, and application of the piezoelectric films to microelectromechanical systems (MEMS) and micrototal-analytical systems (micro-TAS) devices.

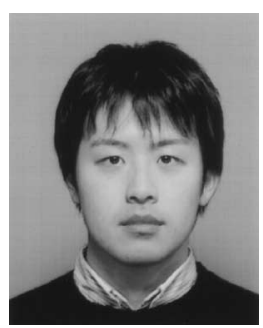

Takaaki Kunisawa was born in Yamaguchi, Japan, in 1980. He received the B.E. degree in mechanical engineering from Nagoya University, Nagoya, Japan, in 2005. He is currently working toward the Master's degree at Kyoto University, Kyoto, Japan. 


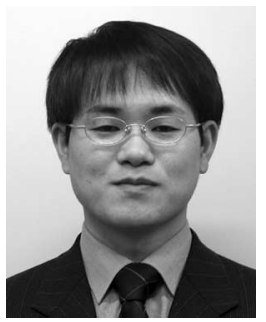

Takaaki Suzuki (S'02-M'03) was born in Gunma, Japan, in 1976. He received the B.E. and M.E. degrees in mechanical system engineering from Gunma University, Maebashi, Japan, in 1998 and 2000, respectively, and the Ph.D. degree in energy science from Kyoto University, Kyoto, Japan, in 2003.

From 2003 to 2004, he was a Research Fellow of the Japan Society for the Promotion of Science. From 2004 to 2005, he was an Assistant Professor in the Department of Mechanical Engineering, Kyoto University, where since 2005, he has been an Assistant Professor in the Department of Micro Engineering. His current research interests include micrototal analysis systems (micro-TAS) and microelectro mechanical systems (MEMS).

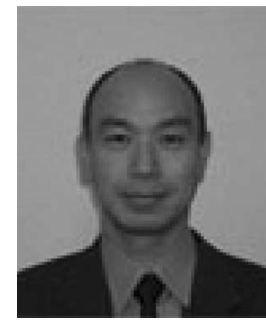

Hidetoshi Kotera was born in Osaka, Japan, in 1957. $\mathrm{He}$ received the B.E., M.E., and Dr.Eng. degrees in mechanical engineering from Kyoto University, Kyoto, Japan, in 1980, 1982, and 1992, respectively.

From 1982 to 1993, he was a Research Manage with Matsushita Electric Industrial Company, Ltd., where he was engaged in numerical analysis of electromagnetics, fluid dynamics, structural analysis, and molecular dynamics at the Central Research Laboratory. From 1993 to 2001, he was an Associate Professor, and from 2001 to 2005 , he was a Professor in the Department of Mechanical Engineering, Kyoto University, where since 2005 , he has been a Professor in the Department of Micro Engineering. His current research interests include microelectromechanical systems (MEMS), micrototal analysis systems (micro-TAS), and computational science. 